\title{
VisMo: Augmented Reality Visualization of Scientific Data and Molecular Structures
}

\author{
Max Collins \\ BWSIP Intern \\ University of Illinois at Urbana- \\ Champaign (Undergraduate) \\ 847-840-1020 \\ macolli2@illinois.edu \\ truecollins@gmail.com
}

\author{
Dr. Alan B. Craig \\ Research Scientist \\ NCSA \\ University of Illinois at Urbana- \\ Champaign \\ a-craig@illinois.edu
}

\begin{abstract}
In this paper, we describe and detail our project that allows for augmented reality visualization of data produced using the Blue Waters supercomputer or other high performance computers. While molecular structures have been displayed using augmented reality before [1][6], we created a pipeline for using information from the Protein Data Bank and automatically loading it into an augmented reality scene for further display and interaction. We find it important to create an easy way for students, scientists, and anyone else to be able to visualize molecular structures using Augmented Reality because it offers an interactive three dimensional perspective that is typically not available in the classroom. Learning about molecular structures in $2 \mathrm{D}$ is much less comprehensive, and our technique for visualization will be free for the end user and offer a great deal of aid to the learning and teaching process. There is no separate purchase required as long as a user has a smart phone or tablet. This is a helpful addition to scientific papers which, if containing the right target image, can be used as the visualization "anchor." The Protein Data Bank (PDB) houses information about proteins, nucleic acids, and more to help scientists and students understand concepts and ideas in biology and chemistry [5]. Our project goal is to open the PDB up to students and people who are not familiar with augmented reality visualization and allow people to learn using the PDB by visualizing molecular structures in different representations, annotating and interacting with the structures, and offering learning modules for common molecular structures. We created a prototype mobile application allowing for molecular visualization of PDB structures, and are continuing to tweak our project for an eventual release to the public.
\end{abstract}

\section{Keywords}

Augmented Reality, Virtual Reality, Blue Waters, Unity 3D, PDB, Molecule, Visualization, Science

Permission to make digital or hard copies of all or part of this work for personal or classroom use is granted without fee provided that copies are not made or distributed for profit or commercial advantage and that copies bear this notice and the full citation on the first page. To copy otherwise, or republish, to post on servers or to redistribute to lists, requires prior specific permission and/or a fee. Copyright OJOCSE, a supported publication of the Shodor Education Foundation Inc. DOI: https://doi.org/10.22369/issn.2153-4136/8/1/3

\section{INTRODUCTION}

"Augmented Reality (AR) is a medium in which digital information is overlaid on the physical world that is in both physical and temporal registration with the physical world and that is interactive in real time," [2]. This is different than virtual reality, which allows us to enter an entirely digital world where our environment is generated by a computer. Our project focus is to allow users to, via a mobile application, display and interact with molecular structures using augmented reality. To do this, we used a game engine, in our case, Unity $3 \mathrm{D}$, so that we did not have to create and develop capabilities that a game engine already has to offer. The game engine allows us to arrange digital information that will be overlaid onto the physical world and thus allow for augmented reality. We also have access to the Blue Waters supercomputer which made it efficient and quick to put structures in the PDB into a format that we could visualize and manipulate. The middle step from PDB to AR visualization is a program called VMD (Visual Molecular Dynamics) which takes structures from the PDB, visualizes them, and creates output in a format that we can make into an Augmented reality scene (a format readable by the game engine software). The end goal is to be able to open the application, which communicates with a server and requests a visualization in the desired format, have the server execute VMD to create a file with the correct specifications and send it back to the mobile application where it is displayed and can be interacted with via AR.

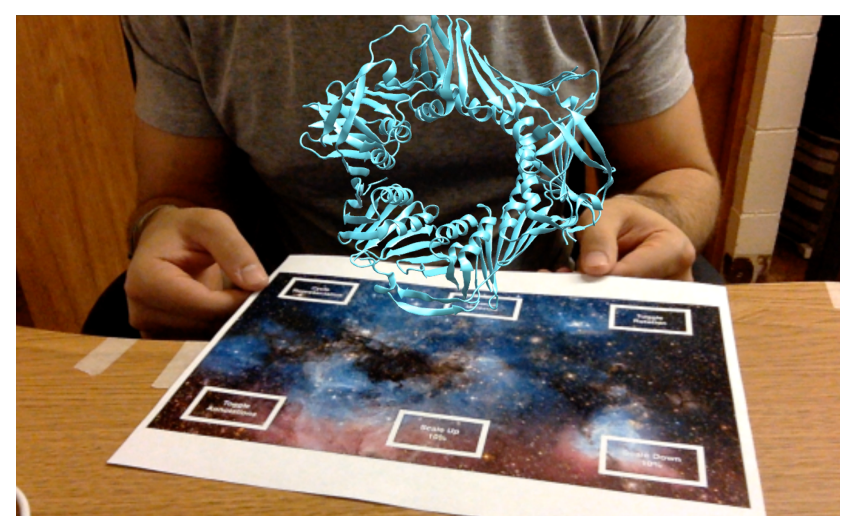

Figure 1: The view as seen from the camera of a computer that is using the VisMo application. You can see the target image, virtual buttons, and molecular structure. 


\section{Related Work}

Work by Billinghurst, Poupyrev, and May about mixed reality environments and how augmented reality allows for collaborative computing is important and relates to concepts we are addressing with in our project [1].

A similar project at the HITLab at the University of Washington for augmented tangible molecular models created a molecular viewer where using virtual models highlighted primary, secondary, tertiary, and quaternary levels of structural organization and amino acid sequences [4].

Another related project is the NCSA Access magazine [5] created by the National Center for Supercomputing Applications (NCSA) at the University of Illinois at Urbana-Champaign. This issue of the magazine was a special augmented reality issue done as collaboration between Dr. Alan Craig, NCSA, and a team at Daqri, a company focused on augmented reality. The pages in the issue had unique content connected with them, and when users view the magazine through the mobile Daqri application, the augmented reality visualizations, including some example molecular visualizations, become visible.

\section{Creating the Pipeline}

\subsection{From PDB to AR}

In order to visualize structures from the PDB, we used Visual Molecular Dynamics (VMD) software. VMD retrieves the data for the requested molecule from the PDB and creates a 3D computer graphics representation of the desired visualization. In our case, VMD creates a .obj file which is then returned to the VisMo application and placed into our virtual scene which is overlaid on the real world via augmented reality. The user of VisMo requests a PDB file within the application by entering the desired molecule via a dialog box on the mobile application, VisMo passes that information along to the server, and VMD runs and produces the desired file with the user-selected specifications. VisMo then can place the visualization in the scene and the user can see and interact it. The user can now control the point of view, size, and other characteristics of the structure they are observing using on screen and virtual buttons.

\subsection{Communicating With a Server}

It was necessary to set up a server for this project because there is computing that must be done outside of the mobile application. Shodor was very helpful and set up a server with VMD on a virtual machine so that we could, from within our augmented reality application, request that a file be made using VMD and certain specifications, and then export the file back to users.

Storing all of the information from the PDB is not feasibly possible within a mobile application, which is why using a server is necessary for this project.

\subsection{Live Annotations}

Creating a system for live annotations within the scene during a visualization session was a goal of ours from the beginning; a way for the viewer to mark up the scene in order to help their comprehension and maybe help them teach a concept to other viewers. For now, we have implemented a "graffiti style" method of annotating the scene in real time. Essentially, when users press a button on the screen, a wall pops up behind the structure and allows the user to draw and write on it, acting as a worksheet of sorts.. In order to do this, we had help from Rodrigo Fernandez who allowed us to work using ideas and modified code from his project Texture Painter which can be found in the Unity 3D asset store [3]. Figure 2 shows a method of graffiti style annotation which could be implemented into the scene.

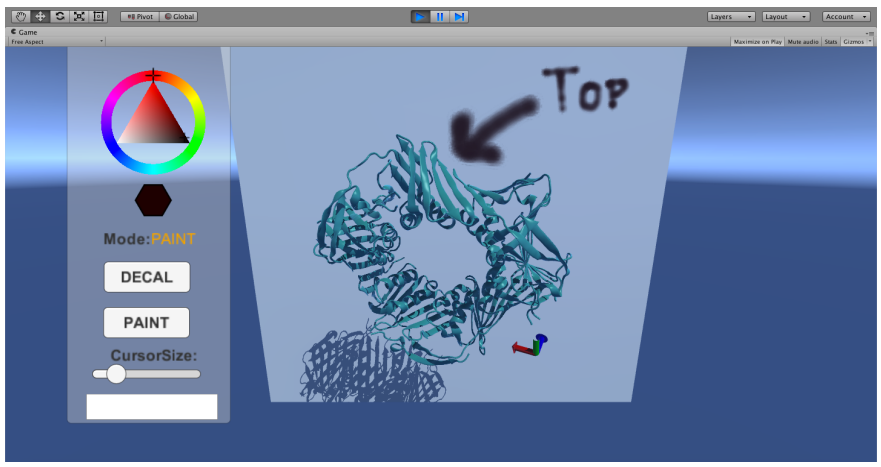

Figure 2: structure of human PCNA with a palette for graffiti style annotations behind it in Unity 3D.

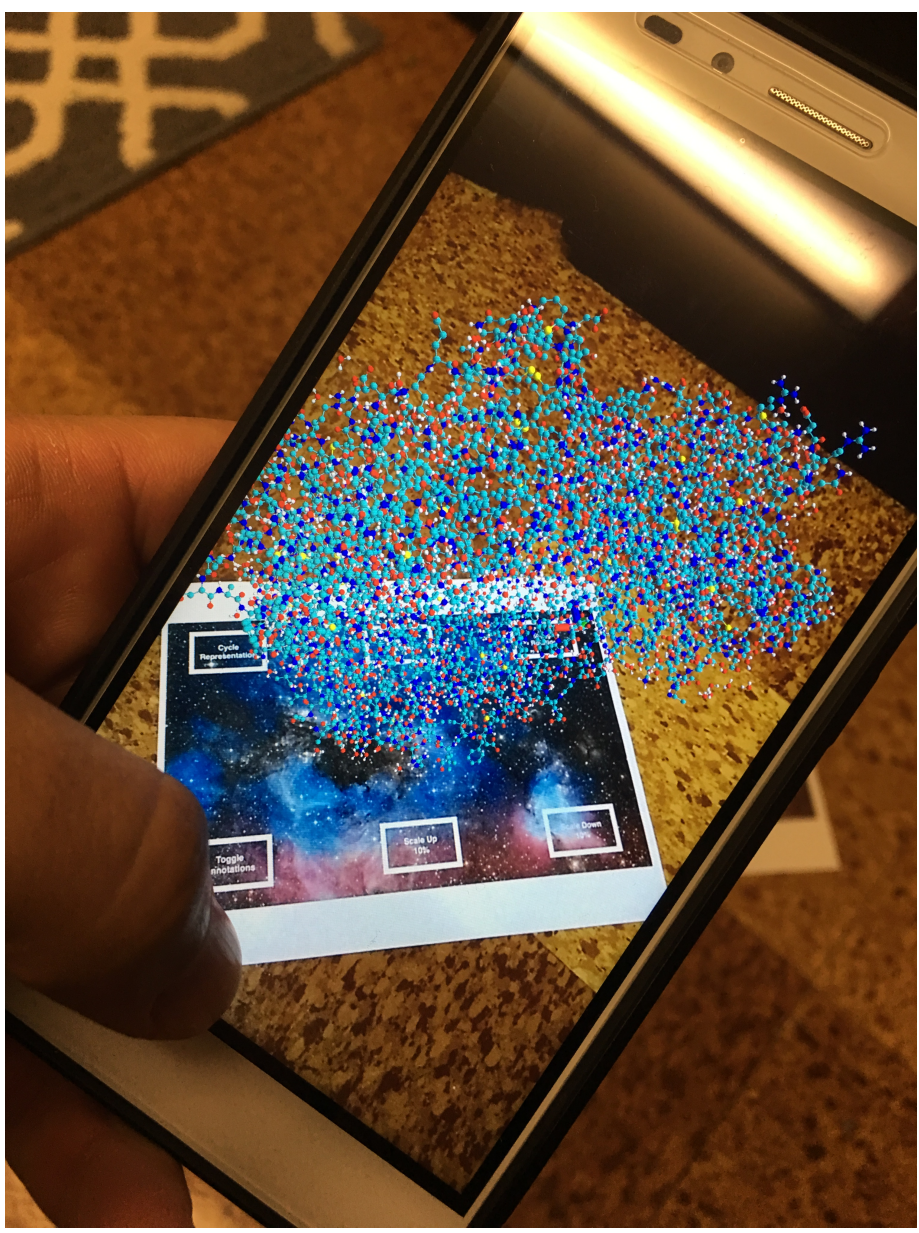

Figure 3: User has the application open in order to visualize a human PCNA structure using the ball and stick visualization technique. 


\section{Application}

The applications for a project like this are vast and relatively unlimited; when we have the ability to control what digital content we are placing in physical and temporal registration with our real world, there is a great deal of versatility.

What we have created is a mobile application that we see as helpful for classroom use, lab use, and other scholarly settings. Our motivation for creating such an application was noticing how limited textbooks and worksheets are when teaching chemistry and biology. Learning about three dimensional structures by looking at them in two dimensional representation simply is not good enough. What we are doing is giving users the ability to manipulate and visualize structures from any angle with no more than a sheet of paper and their smartphone or tablet. It can be seen in Figure 2 that users working with this application will see through the lens of a camera on a tablet or smartphone as their looking glass into the augmented reality world that we have created.

In a lab, scientists often must communicate information with one another, and it is senseless to assume that each and every person they are working with understands exactly what they mean when trying to communicate certain information about certain molecular structures. With our application, scientists can more easily demonstrate concepts by being given the ability to show what they are working with more precisely, and being able to choose representation style, coloring style, etc. to better highlight certain structures or sections of a structure.

Finally, we can say with confidence that readers of scientific articles and papers are often bombarded with heavy text as the only means of information with intermittent pictures scattered throughout the page. Our application would allow users to insert pictures into scientific papers that could be used to display information in augmented reality without compromising the written content of the paper. This would offer a more rounded perspective to information given in a certain paper or article.

\section{Conclusion}

We were able to create an innovative application for Android and iOS platforms which allows users to visualize structures of molecules which are typically only seen in $2 \mathrm{D}$ representations on paper in a 3D space. Our project allows for students and scientists to interact with their work in a way that can foster a greater understanding and sense of comprehension because it is, by nature, more wholesome to our senses. Seeing things from multiple perspectives and multiple representation styles while not being limited to how many physical balls or sticks a classroom has to build a structure is endlessly helpful.

\section{Future Work}

We are continuing to work on this project, and our goal is to familiarize people with augmented reality and allow people to simultaneously enjoy elements of the physical and virtual worlds without shutting out exposure to one or the other at any given time. Viewing digital content and seeing the world around should not be mutually exclusive. We would like to continue to add learning modules into the application which can help people learn scientific concepts and complete tutorials by visualizing scientifically curated structures and reactions using our applications and augmented reality. We will also continue to add features such as expanding our pool of curated structures and working with scientists and teachers to assess what needs we can work to fulfill in the scientific community. We would like to create tutorials where people can learn through pre-designed lessons in addition to the freeform use of visualizing any PDB structure.

\section{Reflections}

The Blue Waters Student Internship Program created an environment where students learned about high performance computing, parallelization, and in general doing research at university level. Students were given the opportunity to sit in on seminars and educational sessions for learning about parallel computing and how to implement ideas from this type of computing into the research and problems that we are working on.

This was a great experience that has molded the education and career path I am taking. As a result of this program, I have already begun taking more computer science courses and visualization courses at University of Illinois at Urbana-Champaign. This has helped me learn more about the basics of computing, and I build upon that more in my independent classes, and I can also tie in the information I have learned from this internship to do what I need to do. This internship has also inspired me to get involved with other departments on campus. I have presented some of my work from this internship at various talks, and as a result of that I have been asked to help with projects and lab studies around campus. I am currently participating in two projects where I am using programming and visualization skills enhanced by this internship.

I plan to do more research at the graduate level as well. I plan to get my $\mathrm{PhD}$ in Informatics or Computer Science, so this internship has definitely had a great influence on me. I now understand how important research is, and I do think that I can make an impact on the scientific community throughout my years of graduate school as I continue to learn and grow more, and participate in more great programs like the Blue Waters Student Internship Program.

\section{ACKNOWLEDGMENTS}

Our thanks to John Stone for helping us with VMD.

Thanks to Mark Van Moer for helping us find the right visualization software and helping and meeting with us as needed.

Thanks to Rodrigo Fernandez for his help with creating a painting unity asset and helping create a custom version for our scene [3].

Thanks to the Blue Waters Student Internship Program for the help and support whenever it was needed. This program gives students a chance to participate in important research and the staff is without question passionate, knowledgable, and driven to allow students in this program to be successful.

This research is part of the Blue Waters sustained-petascale computing project, which is supported by the National Science Foundation (awards OCI-0725070 and ACI-1238993) and the state of Illinois. Blue Waters is a joint effort of the University of Illinois at Urbana-Champaign and its National Center for Supercomputing Applications [7][8][9].

This work used the Extreme Science and Engineering Discovery Environment (XSEDE), which is supported by National Science Foundation grant number ACI-1053575.

Thanks to NASA for supplying the image used as our target image in our figures [10]. This image "Hubble Peers into the Storm" shows a glimpse into the large Magellanic Cloud.

A great thanks to Aaron Weeden for helping with cgi scripting and setting up a server with SHODOR. 


\section{REFERENCES}

1. Billinghurst, M., Poupyrev, I., Kato, H., \& May, R. (n.d.). Mixing realities in Shared Space: An Augmented Reality Interface for Collaborative Computing. 2000 IEEE International Conference on Multimedia and Expo. ICME2000. Proceedings. Latest Advances in the Fast Changing World of Multimedia (Cat. No.00TH8532). doi: 10.1109/icme.2000.871085

2. Craig, A. B. (2013). Understanding Augmented Reality: Concepts and Applications. Morgan Kaufmann.

3. Fernandez, R. (2015). Texture Painting. Retrieved June 22, 2016, from http://codeartist.mx/tutorials/dynamic-texturepainting/

4. HITLab Projects : Augmented Tangible Molecular Models. (n.d.). Retrieved June 23, 2016, from http:// www.hitl.washington.edu/projects/scripps/

5. RCSB Protein Data Bank - RCSB PDB. (n.d.). Retrieved July 09, 2016, from http://www.rcsb.org/pdb/home/home.do

6. University of Illinois: NCSA Access, 25(3), 1-32 . (2012, Fall).

7. Brett Bode, Michelle Butler, Thom Dunning, William Gropp, Torsten Hoe- fler, Wen-mei Hwu, and William Kramer (alphabetical). The Blue Waters Super-System for SuperScience. Contemporary HPC Architectures, Jeffery Vetter editor. Sitka Publications, November 2012.Edited by Jeffrey S . Vetter, Chapman and Hall/CRC 2013, Print ISBN: 978-1-4665-6834-1, eBook ISBN: 978-1-4665-6835-8.

8. Kramer, William, Michelle Butler, Gregory Bauer, Kalyana Chadalavada, Celso Mendes, Blue Waters Parallel I/O Storage Sub-system, High Performance Parallel I/O, Prabhat and Quincey Koziol editors, CRC Publications, Taylor and Francis Group, Boca Raton FL, 2015, Hardback Print ISBN 13:978-1-4665-8234-7.

9. John Towns, Timothy Cockerill, Maytal Dahan, Ian Foster, Kelly Gaither, Andrew Grimshaw, Victor Hazlewood, Scott Lathrop, Dave Lifka, Gregory D. Peterson, Ralph Roskies, J. Ray Scott, Nancy Wilkins-Diehr, "XSEDE: Accelerating Scientific Discovery", Computing in Science \& Engineering, vol.16, no. 5, pp. 62-74, Sept.-Oct. 2014, doi:10.1109/ MCSE.2014.80

10. Hubble Peers into the Storm. (2016, September 9). Retrieved January 16, 2017, from https://www.nasa.gov/image-feature/ goddard/2016/hubble-peers-into-the-storm 